\title{
Conhecimento etnobotânico da Província da Huíla (Angola): um contributo baseado nos registos de campo do colector José Maria Daniel
}

\author{
Francisco Maiato Pedro Gonçalves \\ ISCED Huíla, Departamento de Ciências da Natureza, \\ Herbário do Lubango, Angola \\ Universidade de Hamburgo, Instituto de Ciências Vegetais \\ e Microbiologia, Alemanha
}

\author{
Júlia Elizeth Tchamba \\ Fernanda Maria Oliveira Pires Lages \\ José Luís Mateus Alexandre
}

ISCED Huíla, Departamento de Ciências da Natureza,

Herbário do Lubango, Angola

DOI: https://doi.org/10.31492/2184-2043.RILP2018.35/pp.83-102

\begin{abstract}
Resumo
O presente estudo tem como objetivo divulgar a vida e obra do colector José Maria Daniel, relativamente ao conhecimento etnobotânico da Província da Húla. Extratos da vida e percurso profissional constam da sua auto-biografia. As informações sobre o uso de plantas fazem parte dos cadernos e notas de campo de JMD, encontrados nos arquivos do Herbário do Lubango. Estas informações foram organizadas e introduzidas numa base do Excel, constituindo um total de 787 registos de diversas plantas, correspondentes a 158 espécies, das quais 57 têm uso medicinal, enquanto as restantes foram referenciadas como ornamentais, forrageiras, tóxicas ou venenosas, alimentares e condimentares. Organizou-se a informação conforme a família, género e espécie a que pertencem as plantas, seguida dos usos que lhes são atribuídas e a forma de utilização, constituindo um significativo contributo para a divulgação do conhecimento florístico da região e dos usos das plantas pelas suas populações.
\end{abstract}

Palavras-chave: Herbário do Lubango, José Maria Daniel, plantas medicinais, Província da Huíla.

\begin{abstract}
The present study aims to disclose the life and work of the collector José Maria Daniel, regarding the ethnobotanical knowledge of Huíla Province. Excerpts of his life and professional career are part of his auto-biography, while the information on the use of plants are in notebooks and field notes of JMD found in the archives of the Herbarium of Lubango. This information was organized and introduced in an Excel spreadsheet, constituting a total of 787 records of several plants, corresponding to 158 species, from which 57 are used medicinally, while the remaining were referred as ornamental, forage, toxic or poisonous, food and condiments. These information were organized according to family, genus and species to which the plants belong, following by the uses attributed to them and form of utilization, constituting a significant contribution to the dissemination of the floristic knowledge of the region and the uses of plants by their populations.
\end{abstract}

Keywords: herbarium of Lubango, Huíla province, José Maria Daniel, medicinal plants.

\section{Introdução}

As plantas medicinais são usadas um pouco por todo o mundo e em muitos países constituem muitas vezes o único recurso disponível para as populações, 
principalmente nas áreas rurais. Estima-se que cerca de $80 \%$ da população africana usa a medicina tradicional nos cuidados primários de saúde (WHO, 2003). Os elevados custos dos medicamentos convencionais e dos cuidados de saúde, e a aceitação que os medicamentos tradicionais gozam junto das populações por motivos culturais e espirituais, estão na base do recurso à medicina tradicional (Cunningham, 1988). Angola, com a sua diversidade de ecossistemas que se estende desde as florestas tropicais no norte do país, ao deserto do Namibe a sul (Lovett, 2018), possui uma vasta flora estimada actualmente em 7.076 espécies nativas e 226 espécies introduzidas (Goyder \& Gonçalves, in prep.), e uma rica e transversal medicina tradicional disseminada entre os principais grupos étnicos que, desde cedo, despertou o interesse de vários investigadores (Mendonça et al., 2015). Entretanto, apesar do país ser detentor desta diversidade de plantas e do conhecimento das suas potencialidades na cura de determinadas doenças, apenas uma pequena parte desta informação se encontra disponível para consulta (Costa, 2012).

Isto deve-se ao facto da maior parte dos estudos etnobotânicos feitos no continente africano não terem sido publicados, pois foram realizados por missionários, exploradores e especialistas em várias áreas científicas, como botânicos, médicos e antropólogos, encontrando-se dispersos em arquivos pessoais, bibliotecas, museus e herbários (Liengme, 1983). Estes dados podem ser uma valiosa fonte de informação para o conhecimento da diversidade de plantas e da sua utilização pelas populações. A etnobotânica surge neste contexto como uma área multidisciplinar que permite documentar o saber tradicional, fazendo simultaneamente o reconhecimento da diversidade florística, dando pistas sobre o seu estado de conservação, permitindo desta forma que a potencialidade das plantas como medicinais, bem como os hábitos e costumes das populações que as usam, sejam conhecidos (Costa, 2012).

Devido à grande riqueza cultural do seu povo e à relativa sensação de insegurança durante os longos anos de conflito militar em Angola, grande parte dos estudos etnobotânicos que se conhecem hoje foram realizados na Província da Huíla, localizada a sudoeste de Angola. Com uma extensão territorial de cerca de $78.719 \mathrm{~km}^{2}$, a sua população é actualmente estimada em 2.497 .422 habitantes, distribuída maioritariamente por três grandes grupos étnicos: Nyaneka-humbi, Nganguela e Umbundu (INE, 2016). Do ponto de vista climático, distinguem-se na região duas estações. O inverno, que começa em Maio e se estende até Setembro, é geralmente seco e frio, com temperaturas baixas e ausência completa de chuvas. A estação chuvosa - o verão - vai de Outubro a Abril, é quente, com 
temperaturas médias entre 19 e $21^{\circ} \mathrm{C}$ e precipitação média de 700-1000 mm/ano, sendo o mês de Março o mais chuvoso (Köppen-Geiger, 1936). Para a Província da Húla reconhecem-se oito principais tipos de vegetação (Barbosa 1970). Entretanto estudos recentes apontam para cerca de treze comunidades vegetais e respectivas associações (Chisingui et al., 2018). A província possui um dos mais importantes acervos biológicos do país, entre os quais se destaca o Herbário do Lubango (LUBA), estabelecido na década de 50 do século passado pelo então Instituto de Investigação Científica de Angola (IICA). O herbário, conta actualmente com cerca de 20.000 exemplares e tem como principais colectores Grandvaux Barbosa, Azancot de Menezes, Romeu Santos, Rui Correia e José Maria Daniel (Gonçalves, 2009; Goyder \& Gonçalves in prep.). Associada a estas colecções existe uma vasta informação sobre a ocorrência e uso das plantas na Província da Huíla. Infelizmente as informações sobre a vida e o trabalho de muitos colectores não é fácil de encontrar - não estão disponíveis para a comunidade científica. $\mathrm{O}$ levantamento e análise dos registos destes colectores é de extrema importância, pois permitirá aprofundar os conhecimentos sobre a diversidade florística regional, bem como a disseminação do conhecimento sobre a utilização das plantas locais pela população. Assim, pretende-se com este trabalho divulgar a vida e a obra de José Maria Daniel (JMD), um dedicado explorador e estudioso da flora angolana, com particular realce aos seus trabalhos no domínio da etnobotânica realizados na Província da Huíla.

\section{Materiais \& métodos}

\section{1. Área de estudo}

O presente estudo baseou-se nos registos de campo de JMD, relativamente à utilização das plantas locais pelas populações dos diferentes municípios da província, onde realizou a maior parte do seu trabalho, nomedamente nos municípios do Lubango, Humpata, Chibia e Gambos (Figura 1).

\subsection{Aquisição e tratamento da informação}

Os registos sobre o uso das plantas constam, tal como se referiu acima, dos cadernos e notas de campo de JMD (Figura 2). Estas informações foram compiladas e introduzidas numa base de dados digital em formato MS Excel. Os dados foram organizados de forma hierárquica desde a família, género e espécie, seguida dos usos atribuídos às plantas e a forma da sua utilização. Adicionalmente compilou-se toda a informação das plantas que, não tendo sido referenciadas como medicinais, lhes foram atríbuídas outros usos, como é o caso das plantas 
ornamentais, forrageiras, tóxicas ou venenosas, alimentares e condimentares. A correcção e actualização dos nomes dos taxa foi feita com recurso à mais recente bibliografia fito-taxonómica disponível (Germishuizen et al., 2006; Figueiredo \& Smith, 2008), bem como através da consulta de outras fontes disponíveis online (www.theplantlist.org; www.ipni.org; www.ville-ge.ch). Para uniformizar a nomenclatura, e considerando que parte dos nomes dos taxa estavam desatualizados, recorreu-se à Checklist de Plantas de Angola para os nomes aceites e para os sinónimos (Figueiredo \& Smith, 2008). Os nomes vernáculos na língua nacional Nyaneka e os nomes em português foram escritos com base na literatura existente para o país (Gossweiler, 1953; Figueiredo \& Smith, 2012).

Figura 1. Mapa de Angola e respectiva divisão admistrativa em dezoito províncias. A província da Huíla e os quatro municípios onde se concentraram os trabalhos etnobotânicos do colector estão em destaque.

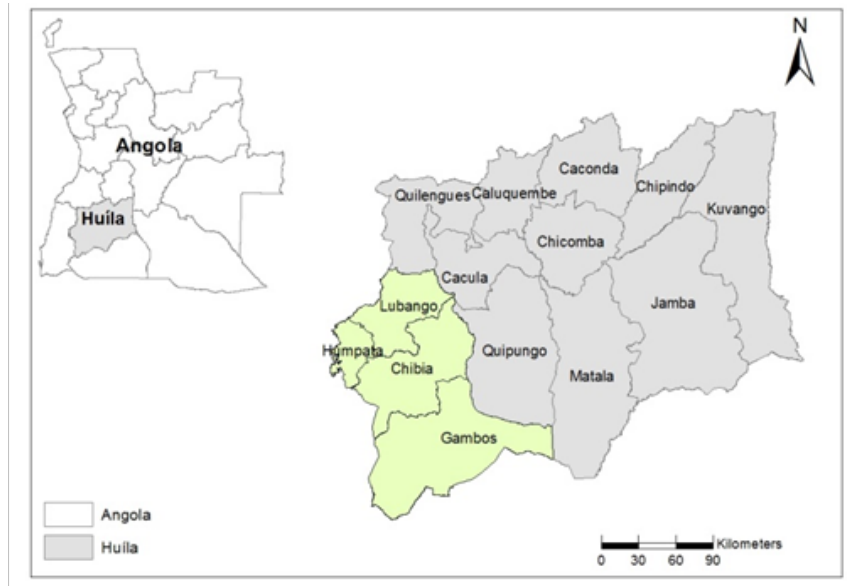

Figura 2. Notas dos cadernos de campo do colector José Maria Daniel, contendo número de colector, nome da planta, nome local e respectiva utilidade.

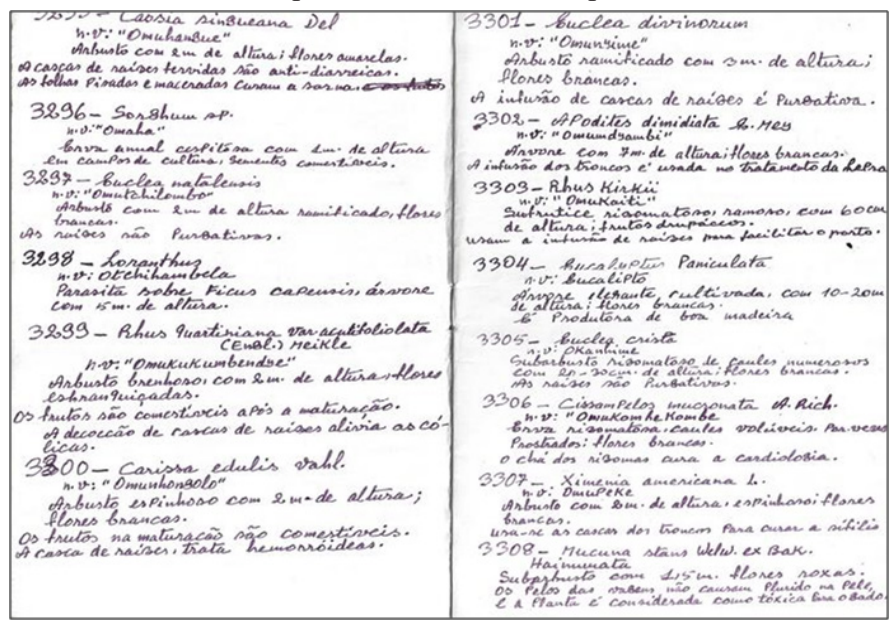




\section{Resultados}

José Maria Daniel (Figura 3) nasceu na Província da Huíla, aos 12 de Setembro de 1943, na Comuna da Huíla, Município do Lubango. Fez os seus estudos primários até a 6 a Classe no Seminário Arquidiocesano do Jau, Missão Católica da Comuna da Huíla. Em Fevereiro de 1964 juntou-se ao extinto IICA, destacado no Centro de Estudos do Lubango, onde permaneceu até Maio de 1967. No ano seguinte foi transferido para Luanda como técnico praticante. Durante este período, JMD beneficiou de várias formações, tendo realizado estudos florísticos com o Eng. Agrónomo Óscar Azancot de Menezes nas Províncias de Benguela, Cuando Cubango, Cunene, Huambo, Huíla, Malange e Namibe. Em 1974 foi nomeado como Técnico na Secção de Plantas da mesma instituição, onde permaneceu até 1994. Durante este período adquiriu competências e prática na conservação de material vegetal, manutenção e gestão de equipamentos de laboratório, combinada com outras competências na área da fito-sistemática. Fez parte de várias expedições botânicas organizadas pela então Secção de Botânica como colector auxiliar, preparando ainda soluções a partir de raízes, folhas de ervas e cascas de troncos de arbustos e de árvores. Participou igualmente de vários inquéritos etnobotânicos conduzidos pela Dra. Manuela Batalha nas províncias do Bengo, Cuanza Sul, Lunda Norte, Lunda Sul, Malange e Moxico.

Figura 3. Colector e ex-curador do Herbário do Lubango José Maria Daniel (1943-2015) com o primeiro autor num trabalho de campo no município da Chibia, província da Huíla em Junho de 2008 (Foto: Ananias José).

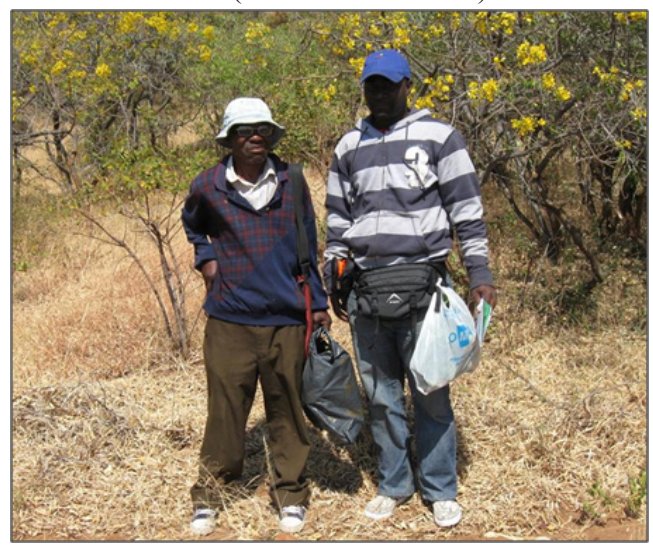

JMD foi um dos mais proeminentes botânicos angolanos após a independência do país em 1975, tendo colhido em quase todas as províncias de Angola. Deixou um acervo de cerca de 3.000 exemplares botânicos todos depositados no Herbário do Lubango (LUBA). Este número poderá ser ainda maior, a julgar pelo enorme quantidade de material depositado no Herbário ainda por avaliar, 
por se desconhecer o número de exemplares depositados noutros herbários do país, como é o caso do Herbário de Luanda (LUA), onde JMD trabalhou durante vários anos, após a extinção do IICA. Em 1987 fez parte dos quadros do Instituto Nacional de Saúde Pública do Lubango (INSPL), colocado no Laboratório do Centro de Estudo de Microbiologia Aplicada, Medicina Tradicional e Divisão Etnobotânica, onde ajudou na organização dos ficheiros de plantas medicinais e preparação de várias soluções. Participou ainda nos inquéritos de etnobotânica e antropologia médica, nas cidades do Lubango e Moçâmedes, tendo entrevistando vários terapeutas tradicionais. A sua vasta experiência com a flora do país, em particular a da Província da Huíla, inclui ainda pesquisas sobre plantas forrageiras, ornamentais, tóxicas ou venenosas, alimentares e condimentares. Com a extinção do INSPL, JMD foi transferido para o Herbário do Lubango, actualmente albergado no ISCED Huíla, onde com dedicação contribuiu para a manutenção e conservação deste importante acervo, numa altura em que o Herbário se encontrava numa situação de quase abandono. Durante este período contribuiu com o seu vasto conhecimento sobre flora angolana para a formação e treino de várias gerações de estudantes angolanos. Passados três anos foi novamente convidado a dar o seu contributo de reorganização do Herbário do Lubango e prestar apoio nos trabalhos de campo no âmbito do Projecto "Angolan Biodiversity Assessment and Capacity Building", promovido por Brian John Huntley, Professor Emérito na Universidade de Cape Town, África do Sul e ex-director do SANBI (Instituto Nacional de Biodiversidade da África do Sul). Infelizmente JMD não concluiu este trabalho, pois após sofrer um acidente vascular cerebral em 2009, a sua saúde deteriorou-se irremediavelmente, acabando por falecer no Hospital Central do Lubango por insuficiência cardio-respiratória. A sua vida inteiramente dedicada à flora angolana, constitui um importante legado para a nova geração de jovens biológos angolanos.

As informações referentes às plantas medicinais, órgãos utilizados, formas de utilização e informações sobre possíveis doenças que curam estão resumidas na Tabela 1. Outras informações contidas nos cadernos e notas de campo de JMD incluem plantas tóxicas ou venenosas, forrageiras, ornamentais, alimentares e também condimentares (Tabela 2). As plantas referidas como medicinais constituem cerca de $33 \%$ do total de espécies registadas, seguida das alimentares $(20 \%)$, ornamentais (18\%), forrageiras (16\%), tóxicas/venenosas (12\%) e com apenas (1\%) as condimentares. A família Fabaceae foi a mais representativa das plantas medicinais, seguida das famílias Vitaceae, Passifloraceae, Lamiaceae, Poaceae. As restantes estão representadas por um único taxon (Figura 4).

Cerca de 787 registos de plantas úteis foram feitos só para a Província Huíla, tendo-se destacado o município do Lubango com maior número de registos 
(86\%), seguindo-se a Humpata (7\%), a Chibia (4\%) e os Gambos (3\%). Para os restantes municípios não foram encontradas quaisquer informações. No total de 67 registos de plantas medicinais foram feitos, 10 registos foram excluídos desta análise por deficiência de informação no que se refere, por exemplo, às partes usadas ou às indicações terapêuticas. O presente estudo contribuiu ainda com novos registos de nomes locais e comuns em Nyaneka e também Português, e que constituem adições à recém publicada lista de nomes comuns da flora angolana. A lista de sinónimos referidos neste estudo encontra-se em anexo e inclui 31 espécies de plantas (Anexo 1).

Figura 4. Percentagem das principais famílias de plantas medicinais do arquivo do colector e ex-curador do Herbário José Maria Daniel.

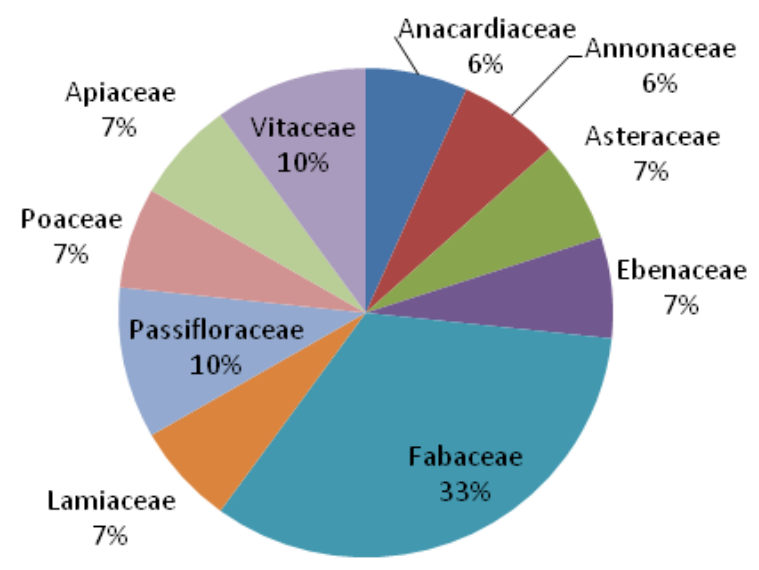

Tabela 1. Principais plantas medicinais documentadas para a Província da Huíla, partes usadas e principais doenças que curam. Estas informações basearam-se nos registos de campo do colector José Maria Daniel. As espécies com o expoente (e) são endémicas da região e $\left(^{*}\right)$ constituem as plantas introduzidas e/ou naturalizadas.

\begin{tabular}{|l|l|l|}
\hline Nome ( científico e Família) & $\begin{array}{l}\text { Forma de utilização e órgãos da } \\
\text { planta utilizados }\end{array}$ & Atributos terapêuticos \\
\hline $\begin{array}{l}\text { Abutilon mauritianum (Jacq.) Me- } \\
\text { dik. (Malvaceae) }\end{array}$ & Decoção das folhas & Emulgente \\
\hline $\begin{array}{l}\text { Adenia lobata (Jacq) Engl. (Passi- } \\
\text { floraceae) }\end{array}$ & Decoção dos rebentos e folhas & Anti-helmíntico \\
\hline $\begin{array}{l}\text { Adenia rumicifolia (Engl \& Harms) } \\
\text { (Passifloraceae) }\end{array}$ & $\begin{array}{l}\text { Decoção da casca do tronco, frutas } \\
\text { comestiveis }\end{array}$ & Fracturas dos membros \\
\hline $\begin{array}{l}\text { Aerva lanata (L) Juss. ex Schult. } \\
\text { (Amaranthaceae) }\end{array}$ & Infusão da planta & Anti-diarreica \\
\hline Blumea gariepina DC. (Asteraceae) & Infusão da planta & Alívio de dores reumáticas \\
\hline $\begin{array}{l}\text { Boerhavia adscendens } \text { Willd. (Nyc- } \\
\text { taginaceae) }\end{array}$ & Decoção do rizoma & Combate de hemorragia \\
\hline
\end{tabular}




\begin{tabular}{|c|c|c|}
\hline $\begin{array}{l}\text { *Brassica nigra Carvalho \& Vas- } \\
\text { concelos (Brassicaceae) }\end{array}$ & Cataplasma da sementes & Reumática \\
\hline $\begin{array}{l}\text { *Cajanus cajan (L.) Millsp. (Faba- } \\
\text { ceae) }\end{array}$ & Decoção das folhas & $\begin{array}{l}\text { Gargarejos e dores de } \\
\text { dentes }\end{array}$ \\
\hline $\begin{array}{l}\text { Canarium schweinfurthii Engl. } \\
\text { (Burseraceae) }\end{array}$ & Resina do tronco & Feridas e úlceras \\
\hline $\begin{array}{l}\text { Cassia angolensis Welw. ex Hiern } \\
\text { (Fabaceae) }\end{array}$ & Infusão da casca do tronco & Desparasitante \\
\hline Catha edulis Forssk. (Celastraceae) & Infusão de folhas secas & $\begin{array}{l}\text { Mastigatório (acção não } \\
\text { especificada) }\end{array}$ \\
\hline $\begin{array}{l}\text { *Chenopodium ambrosioides L. } \\
\text { (Chenopodiaceae) }\end{array}$ & Infusão das folhas & Oxiurose \\
\hline $\begin{array}{l}\text { Cissus aralioides (Welw. Ex Baker) } \\
\text { Planch. (Vitaceae) }\end{array}$ & Raspas da casca do caule & $\begin{array}{l}\text { Coloca-se sobre as feridas } \\
\text { para evitar infecções }\end{array}$ \\
\hline $\begin{array}{l}\text { Cissus rubiginosa (Welw. Ex Baker) } \\
\text { Planch. (Vitaceae) }\end{array}$ & Maceração das folhas & Dores no pescoço \\
\hline Cissus sp. (Vitaceae) & Maceração das folhas & $\begin{array}{l}\text { Esfregaço sobre o corpo } \\
\text { afugenta serpentes }\end{array}$ \\
\hline $\begin{array}{l}{ }^{e} \text { Cochlospermum angolense Welw. } \\
\text { ex Oliv. (Cochlospermaceae) }\end{array}$ & Decoção da raiz & $\begin{array}{l}\text { Hepatites, depurador san- } \\
\text { guíneo }\end{array}$ \\
\hline $\begin{array}{l}\text { Combretum camporum Engl. (Com- } \\
\text { bretaceae) }\end{array}$ & Decoção da casca da raiz & Anti-helmíntico \\
\hline $\begin{array}{l}\text { Crassocephalum multicorymbasum } \\
\text { (Klatt) S.Moore (Asteraceae) }\end{array}$ & Infusão das folhas & $\begin{array}{l}\text { Desinfectante, calmante, } \\
\text { dores reumáticas. }\end{array}$ \\
\hline $\begin{array}{l}\text { Crotalaria amoena Welw. ex Baker } \\
\text { (Fabaceae) }\end{array}$ & Decoção das raizes & Contra retenção urinária \\
\hline $\begin{array}{l}\text { Cymbopogon densiflorus (Steud.) } \\
\text { Stapf. (Poaceae) }\end{array}$ & Decoção das raízes & Prisão de ventre \\
\hline $\begin{array}{l}\text { Cynodon dactylon (L.) Pers. (Po- } \\
\text { aceae) }\end{array}$ & Decoção das raizes & $\begin{array}{l}\text { Diurético e doenças infla- } \\
\text { matórias das vias urinarias }\end{array}$ \\
\hline $\begin{array}{l}\text { *Datura stramonium L. (Solana- } \\
\text { ceae) }\end{array}$ & Maceração das folhas, fumaça & Alívio de dores reumáticas \\
\hline $\begin{array}{l}\text { Dichapetalum lujae De Wild \& T. } \\
\text { Durand (Dichapetalaceae) }\end{array}$ & Decoção da raiz & $\begin{array}{l}\text { Vermes intestinais (lom- } \\
\text { brigas) }\end{array}$ \\
\hline $\begin{array}{l}\text { Diospyros lycioides Desf. (Ebena- } \\
\text { ceae) }\end{array}$ & Decoção das raizes & Purgante \\
\hline $\begin{array}{l}{ }^{e} \text { Dolichos dongaluta Baker (Faba- } \\
\text { ceae) }\end{array}$ & Rizoma & Anginas gangrenosas \\
\hline $\begin{array}{l}\text { Dorstenia psilurus Welw. (Mora- } \\
\text { ceae) }\end{array}$ & Rizoma & $\begin{array}{l}\text { Doenças do aparelho uriná- } \\
\text { rio e dores menstruais }\end{array}$ \\
\hline $\begin{array}{l}\text { Euclea divinorum Hiern (Ebana- } \\
\text { ceae) }\end{array}$ & Decoção da casca da raiz & Purgante \\
\hline Fragaria vesca L. (Rosaceae) & Infusão do rizoma e folhas & Anti-diarreico \\
\hline Garcinia mannii Oliv. (Clusiaceae) & Decoção da semente & Dores estomacais \\
\hline $\begin{array}{l}\text { Hannoa klaineama Pierre ex Engl. } \\
\text { (Scrophulariaceae) }\end{array}$ & Infusão da casca da raiz e do tronco & Cólicas e febres \\
\hline Heeria sp. (Anacardiaceae) & Infusão das folhas & Vómitos \\
\hline
\end{tabular}




\begin{tabular}{|c|c|c|}
\hline $\begin{array}{l}\text { Hymenodictyon floribundum } \\
\text { (Hochst. \& Steud.) Rob. (Rubia- } \\
\text { ceae) }\end{array}$ & Decoção das cascas & Febrífuga e anti-palúdica \\
\hline $\begin{array}{l}\text { Lannea antiscorbutica (Hiern) Engl. } \\
\text { (Anacardiaceae) }\end{array}$ & Casca e entrecasca & $\begin{array}{l}\text { Infecções escorbúticas, } \\
\text { anti-fungico e contração do } \\
\text { canal vaginal }\end{array}$ \\
\hline $\begin{array}{l}\text { Lefebvrea welwitschii Engl. (Apia- } \\
\text { ceae) }\end{array}$ & Planta inteira & Dores toráxicas \\
\hline Lippia asperifolia (Verbenaceae) & Decoção da planta inteira & Banhos puérperais \\
\hline $\begin{array}{l}\text { Lonchocarpus sericeus (Poir) Kunth } \\
\text { ex DC. (Fabaceae) }\end{array}$ & Folhas, casca fresca ou seca e suco & $\begin{array}{l}\text { Actividade microbiana (não } \\
\text { especificada) }\end{array}$ \\
\hline $\begin{array}{l}{ }^{e} \text { Millettia aromatica Dunn. (Faba- } \\
\text { ceae) }\end{array}$ & Fricção do cerne tronco & Cefalalgias reumáticas \\
\hline $\begin{array}{l}\text { Millettia drastica Welw. ex Baker } \\
\text { (Fabaceae) }\end{array}$ & Decoção da semente & Purgante \\
\hline $\begin{array}{l}\text { Mimusops cuneifolia Baker (Sapo- } \\
\text { taceae) }\end{array}$ & Decoção da casca & Prisão de ventre \\
\hline $\begin{array}{l}\text { Monodora myristica (Gaertn.) Du- } \\
\text { nal (Annonaceae) }\end{array}$ & Sementes & $\begin{array}{l}\text { Corrimento e dores esto- } \\
\text { macais }\end{array}$ \\
\hline $\begin{array}{l}\text { Monodora tenuifolia Benth. (Anno- } \\
\text { naceae) }\end{array}$ & Decoção das raizes & $\begin{array}{l}\text { Desinterias e dores de } \\
\text { dentes }\end{array}$ \\
\hline $\begin{array}{l}\text { Mucuna pruriens (L.) DC. var. pru- } \\
\text { riens (Papilionaceae) }\end{array}$ & $\begin{array}{l}\text { Decoção das flores e maceraçao das } \\
\text { raizes }\end{array}$ & $\begin{array}{l}\text { Combate de tosse e cons- } \\
\text { tipações }\end{array}$ \\
\hline $\begin{array}{l}\text { Myrothamnus flabellifolius Welw. } \\
\text { (Myrothamnaceae) }\end{array}$ & Infusão da planta & $\begin{array}{l}\text { Dores lombares, distúrbios } \\
\text { renais, hemorroides, deme- } \\
\text { norreia, tónico energético, } \\
\text { resfiamentos e loção para } \\
\text { escoriações }\end{array}$ \\
\hline Ocimum americanum L. (Labiatae) & Infusão da planta & $\begin{array}{l}\text { Anti-helmíntica e doenças } \\
\text { dos olhos }\end{array}$ \\
\hline Ocimum viride Willd. (Labiatae) & $\begin{array}{l}\text { Infusão das folhas e extremidades } \\
\text { florais }\end{array}$ & $\begin{array}{l}\text { Febrífuga e sudorífica, tóni- } \\
\text { co e calmante }\end{array}$ \\
\hline $\begin{array}{l}\text { Osyris lanceolata Hochst. \& Steud. } \\
\text { (Santalaceae) }\end{array}$ & Infusão da planta inteira & Pneumonia \\
\hline Palisota sp. (Commelinaceae) & Maceração das folhas & Dores intestinais \\
\hline $\begin{array}{l}\text { Parinari curatellifolia } \text { Plach. ex } \\
\text { Benth. (Chrysobalanaceae) }\end{array}$ & Infusão das folhas & Febres altas \\
\hline $\begin{array}{l}\text { Paropsia brazzalana Baill. (Passi- } \\
\text { floraceae) }\end{array}$ & Infusão das folhas & Anti-fungíco, elimina caspa \\
\hline $\begin{array}{l}\text { Peltophorum africanum Sond. (Fa- } \\
\text { baceae) }\end{array}$ & Infusão das raizes & Combate a biliose \\
\hline $\begin{array}{l}\text { Picralima klaineana Welw. ex Hiern } \\
\text { (Apocynaceae) }\end{array}$ & Decoção do fruto & Dores intestinais \\
\hline $\begin{array}{l}\text { Pimpinella platyphylla Welw. (Um- } \\
\text { belliferae) }\end{array}$ & Decoção da raiz & $\begin{array}{l}\text { Usado contra cólicas e } \\
\text { diarreias }\end{array}$ \\
\hline Pistia stratiotes L. (Araceae) & $\begin{array}{l}\text { Cataplasma das folhas misturando } \\
\text { com o leite de coco }\end{array}$ & Tratamento de hemorroides \\
\hline $\begin{array}{l}\text { Plumbago zeylanica L. (Plumbagi- } \\
\text { naceae) }\end{array}$ & Raizes & Lepra e anti-caustica \\
\hline
\end{tabular}




\begin{tabular}{|l|l|l|}
\hline $\begin{array}{l}\text { Ptaeroxylon obliquum (Thumb.) } \\
\text { Radlk (Pteroxylaceae) }\end{array}$ & Decoção da casca do tronco & Anti-paludico \\
\hline $\begin{array}{l}\text { Pterocarpus tinctorius Welw. (Fa- } \\
\text { baceae) }\end{array}$ & Planta inteira & Carminativa \\
\hline $\begin{array}{l}\text { Spathodea companulata Beauv. } \\
\text { (Bignoniaceae) }\end{array}$ & Flores & $\begin{array}{l}\text { Tratamento de chagas e } \\
\text { feridas ulcerosas }\end{array}$ \\
\hline
\end{tabular}

Tabela 2. Nomes locais em Nyaneka (Nya) e em Português (PT) dos principais taxa, e respectivas famílias referidos no presente estudo como ornamentais (Or), forrageiras (Fo), alimentares $(\mathrm{Al})$, tóxicas ou venenosas $(\mathrm{To})$ e condimentares $(\mathrm{Co}) .\left(^{*}\right)$ Plantas introduzidas e/ou naturalizadas.

\begin{tabular}{|c|c|c|c|c|c|c|c|}
\hline Nome científico (Família) & Nome local (Nya) & $\begin{array}{l}\text { Nome local } \\
\text { (PT) }\end{array}$ & Or & Fo & Al & To & Co \\
\hline $\begin{array}{l}\text { Acacia brevispica Harms (Fa- } \\
\text { baceae) }\end{array}$ & Olukienye & & & $\sqrt{ }$ & & & \\
\hline $\begin{array}{l}\text { Acacia tristis (Welw. ex Oliv.) } \\
\text { A.Schreib. (Fabaceae) }\end{array}$ & Omunkhondo & & & & $\sqrt{ }$ & & \\
\hline $\begin{array}{l}\text { Adenodolichos huillensis Torre } \\
\text { var. huillensis (Fabaceae) }\end{array}$ & Omanhingua & & & & $\sqrt{ }$ & & \\
\hline $\begin{array}{l}\text { *Agave sisalana Perrine (Aga- } \\
\text { vaceae) }\end{array}$ & & Sisal & & & & $\sqrt{ }$ & \\
\hline $\begin{array}{l}\text { Albizia adianthifolia (Schuma- } \\
\text { ch.) W.Wight var. intermedia } \\
\text { (Fabaceae) }\end{array}$ & & & & & & $\sqrt{ }$ & \\
\hline $\begin{array}{l}\text { Albizia coriaria Wel. ex Oliv. } \\
\text { (Fabaceae) }\end{array}$ & & & & & & $\sqrt{ }$ & \\
\hline $\begin{array}{l}\text { *Amaranthus caudatus L. (Ama- } \\
\text { ranthaceae) }\end{array}$ & Omboa & $\begin{array}{l}\text { Chorões-dos- } \\
\text {-jardins }\end{array}$ & & & $\sqrt{ }$ & & \\
\hline $\begin{array}{l}\text { Amaranthus lividus L. (Amaran- } \\
\text { thaceae) }\end{array}$ & Omboa & & & & $\sqrt{ }$ & & \\
\hline Amaranthus sp. (Amaranthaceae) & Omboa & & & & $\sqrt{ }$ & & \\
\hline $\begin{array}{l}\text { Amblygonacarpus obtusangulus } \\
\text { (Oliv.) Harms }\end{array}$ & & & & & & $\sqrt{ }$ & \\
\hline Ancylanthos sp. (Rubiaceae) & Enngen & & & & $\sqrt{ }$ & & \\
\hline $\begin{array}{l}\text { Andropogon gayanus Kunth. } \\
\text { (Poaceae) }\end{array}$ & Kota-kota & & & $\sqrt{ }$ & & & \\
\hline $\begin{array}{l}\text { Annona muricata L. (Annona- } \\
\text { ceae) }\end{array}$ & & Fruta-pinha & $\sqrt{ }$ & & & & \\
\hline $\begin{array}{l}\text { Bauhinia tomentosa L. (Faba- } \\
\text { ceae) }\end{array}$ & Otyengangu & & $\sqrt{ }$ & & & & \\
\hline $\begin{array}{l}\text { Bidens biternata (Lour.) Merr. \& } \\
\text { Shuff. (Asteraceae) }\end{array}$ & Omukolokoho & & & & $\sqrt{ }$ & & \\
\hline $\begin{array}{l}\text { Bobgunnia madagascariensis } \\
\text { (Desv.) J.H.Kirkbr. \& Wiersema } \\
\text { (Fabaceae) }\end{array}$ & Omuhombo & Pau-rosa & & & & $\sqrt{ }$ & \\
\hline $\begin{array}{l}\text { *Bougainvillea glabra } \text { Choisy } \\
\text { (Nyctaginaceae) }\end{array}$ & & Buganvília & $\sqrt{ }$ & & & & \\
\hline
\end{tabular}




\begin{tabular}{|c|c|c|c|c|c|c|c|}
\hline $\begin{array}{l}\text { Brachiaria brizantha (A.Rich.) } \\
\text { Stapf. (Poaceae) }\end{array}$ & & Capim-ocinde & & $\sqrt{ }$ & & & \\
\hline Brachystegia sp. (Fabaceae) & & & & & $\sqrt{ }$ & & \\
\hline *Canna indica L. (Cannaceae) & & Cana-da-Índia & $\sqrt{ }$ & & & & \\
\hline $\begin{array}{l}\text { Capsicum annuиm L. (Solana- } \\
\text { ceae) }\end{array}$ & Omundungu & $\begin{array}{l}\text { Pimententeira- } \\
\text {-jindungo }\end{array}$ & & & & & $\sqrt{ }$ \\
\hline *Capsicum sp. (Solanaceae) & Okatiata & & & & & & $\sqrt{ }$ \\
\hline $\begin{array}{l}\text { Carissa edulis (Forssk.) Vahl } \\
\text { (Apocynaceae) }\end{array}$ & Omunhongolo & & & & $\sqrt{ }$ & & \\
\hline Cassia fistula L. (Fabaceae) & & Cana-fistula & $\sqrt{ }$ & & & & \\
\hline $\begin{array}{l}\text { Chrysophyllum argyrophyllum } \\
\text { Hiern (Sapotaceae) }\end{array}$ & Omutomboti & & & & $\sqrt{ }$ & & \\
\hline $\begin{array}{l}\text { Clematis chrysocarpa Welw. ex } \\
\text { Oliv. (Ranculaceae) }\end{array}$ & Epeke-peke & & $\sqrt{ }$ & & & & \\
\hline $\begin{array}{l}\text { Colophospermum mopane (J. } \\
\text { Kirk ex Benth.) J.Léonard (Faba- } \\
\text { ceae) }\end{array}$ & Omuntiati & & & $\sqrt{ }$ & & & \\
\hline $\begin{array}{l}\text { Conyza gouanii (L.) Willd. (As- } \\
\text { teraceae) }\end{array}$ & Ombambambilingui & & $\sqrt{ }$ & & & & \\
\hline $\begin{array}{l}\text { *Cosmos bipinnatus Cav. (As- } \\
\text { teraceae) }\end{array}$ & & Cosmea & $\sqrt{ }$ & & & & \\
\hline $\begin{array}{l}\text { Cynodon dactylon (L.) Pers. } \\
\text { (Poaceae) }\end{array}$ & Otyiwena & Escalrracho & & $\sqrt{ }$ & & & \\
\hline $\begin{array}{l}\text { *Dianthus caryophyllus L. } \\
\text { (Caryophyllaceae) }\end{array}$ & & Craveiro & $\sqrt{ }$ & & & & \\
\hline $\begin{array}{l}\text { Dichrostachys glomerata (Fors- } \\
\text { sk.) Chiov. (Fabaceae) }\end{array}$ & Muhaniambungu & & & $\sqrt{ }$ & & & \\
\hline $\begin{array}{l}\text { Dioscorea quartiniana A.Rich. } \\
\text { (Dioscoreaceae) }\end{array}$ & & & & & & $\sqrt{ }$ & \\
\hline *Dahlia rosea Cav. (Astercaeae) & & Dálias & $\sqrt{ }$ & & & & \\
\hline $\begin{array}{l}\text { Echinochloa pyramidalis (Lam.) } \\
\text { Hutch. \& Chase (Poaceae) }\end{array}$ & & & & $\sqrt{ }$ & & & \\
\hline $\begin{array}{l}\text { Eragrostis racemosa (Tumb) } \\
\text { Steud. (Poaceae) }\end{array}$ & & & & $\sqrt{ }$ & & & \\
\hline $\begin{array}{l}\text { Eriospermum flexuosum Welw. } \\
\text { ex Baker (Eriospermaceae) }\end{array}$ & $\begin{array}{l}\text { Otchilombango- } \\
\text { longo }\end{array}$ & & & & & $\sqrt{ }$ & \\
\hline $\begin{array}{l}\text { Euphorbia rhipsaloides Welw. } \\
\text { (Euphorbiaceae) }\end{array}$ & & Almeidinha & $\sqrt{ }$ & & & & \\
\hline $\begin{array}{l}\text { Fadogia monticola Robyns (Ru- } \\
\text { biaceaceae) }\end{array}$ & Otyiningui & & & & $\sqrt{ }$ & & \\
\hline Ficus elastica Roxb. (Moraceae) & Omulemba & $\begin{array}{l}\text { Figueira-da-bor- } \\
\text { racha }\end{array}$ & & & & $\sqrt{ }$ & \\
\hline $\begin{array}{l}\text { Ficus gnaphalocarpa (Miq.) } \\
\text { Steud. ex Miq. (Moraceae) }\end{array}$ & Omukuyumbua & & & $\sqrt{ }$ & $\sqrt{ }$ & & \\
\hline Ficus petersii Warb. (Moraceae) & Omuholo & & & & $\sqrt{ }$ & & \\
\hline $\begin{array}{l}\text { Ficus pygmaea Welw.ex Hiern } \\
\text { (Moraceae) }\end{array}$ & Otchikuiu & & & & $\sqrt{ }$ & & \\
\hline
\end{tabular}




\begin{tabular}{|c|c|c|c|c|c|c|}
\hline $\begin{array}{l}\text { Ficus welwitschii Warb. (Mo- } \\
\text { raceae) }\end{array}$ & Omulemba-mbuja & & & $\sqrt{ }$ & & \\
\hline $\begin{array}{l}\text { Gerardiina angolensis Engl. } \\
\text { (Orobanchaceae) }\end{array}$ & & & $\sqrt{ }$ & & & \\
\hline $\begin{array}{l}\text { Gnidia chrysantha Gilg. } \\
\text { (Thymaelaceae) }\end{array}$ & & & $\sqrt{ }$ & & & \\
\hline $\begin{array}{l}\text { *Gomphrena globosa L. (Ama- } \\
\text { ranthaceae) }\end{array}$ & & Perpêtuas-roxas & $\sqrt{ }$ & & & \\
\hline $\begin{array}{l}\text { Heeria argyrocrysea Engl \& } \\
\text { Gilg. (Asteraceae) }\end{array}$ & & & & & $\sqrt{ }$ & \\
\hline $\begin{array}{l}\text { Helichrysum steetzii (Vatke) } \\
\text { O.Hoffm. (Asteraceae) }\end{array}$ & Oluhiti & & $\sqrt{ }$ & & & \\
\hline $\begin{array}{l}\text { Heteropogon contortus (L.) } \\
\text { Roem. \& Schuet. (Poaceae) }\end{array}$ & Capiongo & Capim-osoke & & $\sqrt{ }$ & & \\
\hline $\begin{array}{l}\text { Hyparrhenia familiaris (Steud.) } \\
\text { Stapf. (Poaceae) }\end{array}$ & & & & $\sqrt{ }$ & & \\
\hline $\begin{array}{l}\text { *Ipomoea batatas (L.) Lam. } \\
\text { (Convolvulaceae) }\end{array}$ & & Batata-doce & & & $\sqrt{ }$ & \\
\hline $\begin{array}{l}\text { *Jacaranda mimosifolia Dow. } \\
\text { (Fabaceae) }\end{array}$ & & Jacarandá & $\sqrt{ }$ & & & \\
\hline $\begin{array}{l}\text { *Lantana camara L. (Verbena- } \\
\text { ceae) }\end{array}$ & & Lantano & & & $\sqrt{ }$ & \\
\hline $\begin{array}{l}\text { *Leucaena glauca Benth. (Faba- } \\
\text { ceae) (sin. Leucaena leucocepha- } \\
\text { la (Lam.) De Wit.) }\end{array}$ & & Lantoro & & $\sqrt{ }$ & & \\
\hline $\begin{array}{l}\text { *Ligustrum japonicum Thumb. } \\
\text { (Oleaceae) }\end{array}$ & & $\begin{array}{l}\text { Alfenheiro-do- } \\
\text {-Japão }\end{array}$ & $\sqrt{ }$ & & & \\
\hline $\begin{array}{l}\text { Loudetia simplex (Nees) } \\
\text { C.E.Hubb. (Poaceae) }\end{array}$ & & & & $\sqrt{ }$ & & \\
\hline *Melia azedarach L. (Meliaceae) & & Amargoseira & $\sqrt{ }$ & & & \\
\hline $\begin{array}{l}\text { Melinis minutiflora P. Beauv. } \\
\text { (Poaceae) }\end{array}$ & & Capim-gordura & & $\sqrt{ }$ & & \\
\hline $\begin{array}{l}\text { *Mirabilis jalapa L. (Nyctagy- } \\
\text { naceae) }\end{array}$ & & $\begin{array}{l}\text { Maravilha-do- } \\
\text {-Perú }\end{array}$ & $\sqrt{ }$ & & & \\
\hline $\begin{array}{l}\text { *Morus alba L. var. multicaulis } \\
\text { (Perr.) Loudon (Moraceae) }\end{array}$ & & $\begin{array}{l}\text { Amoreira-bran- } \\
\text { ca }\end{array}$ & $\sqrt{ }$ & & & \\
\hline $\begin{array}{l}\text { Mucuna stans Welw. ex Baker } \\
\text { (Fabaceae) }\end{array}$ & Hainumata & Feijão-maluco & & & & $\sqrt{ }$ \\
\hline $\begin{array}{l}\text { Mundulea sericea (Willd.) A. } \\
\text { Chev. (Fabaceae) }\end{array}$ & Onkongo & & & & & $\sqrt{ }$ \\
\hline $\begin{array}{l}\text { *Nasturtium offcinale R.Br. } \\
\text { (Brassicaceae) }\end{array}$ & & Agrião-de-água & & & $\sqrt{ }$ & \\
\hline *Nasturtium sp.( Brassicaceae) & & Agrião & & & $\sqrt{ }$ & \\
\hline $\begin{array}{l}\text { *Nerium oleander L. (Apocy- } \\
\text { naceae) }\end{array}$ & & Cevadilha & $\sqrt{ }$ & & & $\sqrt{ }$ \\
\hline $\begin{array}{l}\text { Parinari curatellifolia } \text { Planch. ex } \\
\text { Benth. (Chrysobalanaceae) }\end{array}$ & Ontyahi & & & & $\sqrt{ }$ & \\
\hline
\end{tabular}




\begin{tabular}{|c|c|c|c|c|c|c|}
\hline $\begin{array}{l}\text { *Parkinsonia aculeata L. (Fa- } \\
\text { baceae) }\end{array}$ & & $\begin{array}{l}\text { Espinheiro-de- } \\
\text {-Jerusalém }\end{array}$ & $\sqrt{ }$ & & & $\sqrt{ }$ \\
\hline $\begin{array}{l}\text { Peucedanum angolense (Welw. } \\
\text { ex Ficalho) Cannon (Apiaceae) }\end{array}$ & Ondunda & & & & $\sqrt{ }$ & \\
\hline $\begin{array}{l}\text { Phragmites mauritianus Kunth. } \\
\text { (Poaceae) }\end{array}$ & Humbu & Caniço & & $\sqrt{ }$ & & \\
\hline $\begin{array}{l}\text { *Physalis peruviana L. (Sola- } \\
\text { naceae) }\end{array}$ & Omutipatipa & & & & $\sqrt{ }$ & \\
\hline $\begin{array}{l}\text { Phytolacca dodecandra L'Hér. } \\
\text { (Phytolaccaceae }\end{array}$ & & & & & & $\sqrt{ }$ \\
\hline *Pimpinella anysum (Apiaceae) & & Erva-doce & & & $\sqrt{ }$ & \\
\hline *Populus alba L. (Salicaceae) & & Choupo & $\sqrt{ }$ & & & \\
\hline $\begin{array}{l}\text { Psorospermum tenuifolium } \\
\text { Hook.f. (Hypericaceae) }\end{array}$ & & & & & & $\sqrt{ }$ \\
\hline $\begin{array}{l}\text { Pteleopsis anisoptera (Welw. } \\
\text { ex M.A.Lawson) Engl \& Diels } \\
\text { (Combretaceae) }\end{array}$ & Omuhihi & & & & $\sqrt{ }$ & \\
\hline $\begin{array}{l}\text { *Raphanus raphanistrum } \mathrm{L} . \\
\text { (Brassicaceae) }\end{array}$ & & $\begin{array}{l}\text { Rabanete; Cou- } \\
\text { ve-nabo }\end{array}$ & & & $\sqrt{ }$ & \\
\hline $\begin{array}{l}\text { Rhus natalensis Bernh. (Anacar- } \\
\text { diaceae) }\end{array}$ & Omukukumbendye & & & & $\sqrt{ }$ & \\
\hline Rhus sp. (Anacardiaceae) & & & & & $\sqrt{ }$ & \\
\hline $\begin{array}{l}\text { Rhynchelytrum repens (Willd.) } \\
\text { C.E.Hubb. (Poaceae) }\end{array}$ & Onkhulungumbe & Capim-favorita & & $\sqrt{ }$ & & \\
\hline *Rosa spp. (Rosaceae) & & Roseira & $\sqrt{ }$ & & & \\
\hline *Sambucus nigra L. (Adoxaceae) & & Sabugueiro & $\sqrt{ }$ & & & \\
\hline $\begin{array}{l}\text { Setaria flabellata Stapf. (Poa- } \\
\text { ceae) }\end{array}$ & & & & $\sqrt{ }$ & & \\
\hline Sida alba L. (Malvaceae) & & & & $\sqrt{ }$ & & $\sqrt{ }$ \\
\hline $\begin{array}{l}\text { Sorghum cafrorum (Retz.) } \\
\text { P.Beauv. (Poaceae) }\end{array}$ & Oinaha & Massambala & & & $\sqrt{ }$ & \\
\hline Sorghum sp. (Poaceae) & & Massambala & & & $\sqrt{ }$ & \\
\hline Sorghum sp. (Poaceae) & Omuha & & & & $\sqrt{ }$ & \\
\hline $\begin{array}{l}\text { Strychnos aculeata Soler (Stry- } \\
\text { chnaceae) }\end{array}$ & & & & & & $\sqrt{ }$ \\
\hline Stylosanthes sp. (Fabaceae) & & & & $\sqrt{ }$ & & \\
\hline *Tagetes erecta L. (Asteraceae) & & Cravo-da-Índia & $\sqrt{ }$ & & & \\
\hline $\begin{array}{l}\text { Tarchonanthus camphoratus L. } \\
\text { (Asteraceae) }\end{array}$ & Omukando & $\begin{array}{l}\text { Pau-quicongo- } \\
\text {-da-Huíla }\end{array}$ & & $\sqrt{ }$ & & \\
\hline $\begin{array}{l}\text { Tephrosia cephalantha Welw. ex } \\
\text { Baker (Fabaceae) }\end{array}$ & & & & & & $\sqrt{ }$ \\
\hline $\begin{array}{l}\text { Tephrosia vogelii Hook.f. (Fa- } \\
\text { baceae) }\end{array}$ & Kadia-sasa & Mata-peixe & & & & $\sqrt{ }$ \\
\hline $\begin{array}{l}\text { Tetrapleura tetraptera (Schuma- } \\
\text { ch. \& Thonn.)Taub. (Fabaceae) }\end{array}$ & & & & & & $\sqrt{ }$ \\
\hline
\end{tabular}




\begin{tabular}{|l|l|l|l|l|l|l|l|}
\hline $\begin{array}{l}\text { *Thevetia peruviana (Pers.) } \\
\text { Schum. (Apocynaceae) }\end{array}$ & $\begin{array}{l}\text { Chapéu-de-Na- } \\
\text { poleão }\end{array}$ & $\sqrt{ }$ & & & & \\
\hline $\begin{array}{l}* \text { Thuja orientalis L. (Cupressa- } \\
\text { ceae) }\end{array}$ & Árvore-da-vida & $\sqrt{ }$ & & & & \\
\hline $\begin{array}{l}\text { Vernonia gerberiformis Oliv. \& } \\
\text { Hiern subsp. macrocyanus (O. } \\
\text { Hoffm.) C.Jeffrey (Asteraceae) }\end{array}$ & Ekakaholua & & & & & $\sqrt{ }$ & \\
\hline $\begin{array}{l}\text { Vetiveria nigritana (Benth.) Sta- } \\
\text { pf. (Poaceae) }\end{array}$ & & & & $\sqrt{ }$ & & & \\
\hline $\begin{array}{l}\text { *Vigna unguiculata (L.) Walp. } \\
\text { (Fabaceae) }\end{array}$ & Macunde & Feijão-frade & & & $\sqrt{ }$ & & \\
\hline *Zea mays L. (Poaceae) & & Milho & & & $\sqrt{ }$ & & \\
\hline $\begin{array}{l}\text { *Zinnia elegans Jacq. (Astera- } \\
\text { ceae) }\end{array}$ & & Zínia & $\sqrt{ }$ & & & & \\
\hline
\end{tabular}

\section{Discussão}

Angola é um país com com imensa diversidade biológica que desde cedo despertou o interesse de colectores botânicos, como Friedrich Welwitsch, Hugo Baum e John Gossweiler que se destacaram pela importância das suas coleções (Figueiredo \& Smith, 2008; Figueiredo et al., 2009). Existem, entretanto, muitos outros colectores que deram o seu contributo para o conhecimento da flora do país e da Província da Huíla em particular (Gonçalves, 2009). Encontrar informação sobre estes colectores não é uma tarefa fácil, pois os dados sobre a sua vida e obra encontram-se dispersos por várias fontes e, muitas vezes, publicados em diferentes línguas (Figueiredo \& Smith, 2008). A vida e obra dos colectores nacionais é ainda mais difícil de investigar, porque muitos deles trabalharam apenas como auxiliares dos principais colectores da época, não tendo sido referidos nem devidamente reconhecidos no que se refere ao espólio que deixaram e no que produziram para o conhecimento da flora angolana. Poucos são os colectores nacionais que se destacaram no período pós-independência de Angola, uma vez que o país mergulhou numa guerra civil, que destruiu as infraestruturas que existiam na altura, relegando a investigação científica para segundo plano.

O conhecimento etnobotânico de Angola é escasso e encontra-se restrito ao conhecimento tradicional sendo, no entanto, reconhecido o seu valor social, cultural e também espiritual. Apesar do poder de cura que é atribuído às plantas, muito pouco tem sido feito relativamente ao respectivo conhecimento químico. Para o conhecimento etnobotânico de Angola podem-se destacar os trabalhos pioneiros de Brito Teixeira (1960), Óscar Azancot de Menezes (unpub.) e Manuela Batalha Van-Dúnem (1979; 1994) cujos resultados não estão adequadamente explorados. Mais tarde, destacaram-se os trabalhos de 
Bossard (1996) sobre a medicina tradicional no Centro e Oeste de Angola, de Rosa Melo (2008) sobre plantas medicinais dos Handas do Sul de Angola e, mais recentemente, um estudo de Raquel Kissanga (2006) sobre a valorização da flora e utilização das plantas lenhosas como combustível e na construção no planalto central e sudeste de Angola.

O trabalho de José Maria Daniel tem um elevado valor para a divulgação do conhecimento da flora da Província da Huíla, bem como da sua utilização pela população local, com destaque para quatro municípios, nomedamente Lubango, Humpata, Chibia e Gambos. A ausência de informação florística referente aos restantes municípios deve-se, provavelmente, às dificuldades de acesso a estas áreas durante o período em que o colector trabalhou, e pela insegurança que caracterizou muitas regiões do país. Grande parte das plantas referidas neste estudo como medicinais, encontram-se igualmente referidas em vários estudos desenvolvidos no país e noutras regiões, com ênfase à parte austral do continente africano. Os órgãos utilizados, bem como as respectivas aplicações variam muito de região para região. Estudos realizados em Angola indicam a utilização das raízes carnosas de Cochlospemum angolense no tratamento preventivo de icterícia e de biliosas, além de ser um excelente diurético; Lippia asperifolia cuja infusão das folhas é utilizada no tratamento de pontadas e dores de peito provocadas, possivelmente por constipações, enquanto a infusão das folhas e inflorescências de Cymbopogon densiflorus está referida como sendo um eficaz febrífugo, em constipações e possivelmente pneumonias (Teixeira, 1960). Num outro estudo realizado no Zimbabwe algumas das plantas referidas no nosso estudo como medicinais, alimentares e forrageiras são assinaladas com outras aplicações, como acontece, por exemplo, com Carissa edulis (=Carissa spinarum), Parinari curatellifolia, Diospyros lycioides, Peltophorum africanum, Lantana camara e Lippia asperifolia (Maroyi, 2013), algumas espécies como Colophospermum mopane, Cynodon dactylon e Ficus sycomorus (Ficus gnaphalocarpa), referidas apenas como forrageiras por JMD, são atribuídos outros atributos, assim como é o caso de C. mopane cujo produto resultante da infusão das folhas, cascas do tronco e raíz moída, é utilizado no tratamento de disenterias, alívio de dores estomacais e no tratamento de doenças venéreas como a sífilis (Ribeiro et al., 2010; Chinsembu, 2016).

Ficus sycomorus cujos frutos são comestíveis, é utilizado no tratamento de micoses e de outras doenças dermatológicas, enquanto às folhas de Cynodon dactylon são atribuídas propriedades ginecológicas (Ribeiro et al., 2010). Diospyros lyciodes, Euclea divinorum, Peltophorum africanum e Plumbago zeylanica foram igualmente referidas no tratamento de várias infecções de transmissão sexual, num 
estudo recente realizado na Zâmbia (Chinsembu, 2016). Pelo contrário este estudo é coincidente com as aplicações atribuídas à espécie Myrothamnus flabellifolius, no que se refere às múltiplas utilidades da planta, e com Hymenodictyon floribun$d u m$, pelas propriedades anti-palúdicas da casca deste arbusto, comum nas regiões montanhosas do planalto da Huíla (Bossard, 1996).

As plantas forrageiras e tóxicas principalmente para o gado, constituem outra importante fonte de informação do colector. Esta vasta lista, inclui herbáceas como Sida alba e várias gramíneas como Mellinis minutiflora, Brachyaria brizantha, Heteropogon contortus e Andropogon gayanus. Algumas destas espécies foram referidas como forrageiras de grande valor nutricional para o gado, num estudo realizado entre as comunidades pastoris da Bibala, Cahama e Gambos, municípios pertencentes às Províncias do Namibe, Cunene e Huíla, respectivamente (Maiato \& Sweet, 2011). As plantas tóxicas ou venenosas incluem várias espécies da flora local como Bobgunnia madagascariensis, Mucuna stans, Mundulea sericea entre outras, e espécies introduzidas como Nerium oleander, Parkinsonia aculeata e Agave sisalana, esta última cultivada em larga escala em quase toda a África austral para fins industriais (Walters et al., 2011).

Outras espécies que não fazem parte da flora nativa foram introduzidas devido provavelmente ao seu valor nutricional, medicinal ou mesmo ornamental, tornando-se naturalizadas. Assim, Lantana camara e outras da flora local como Parinari curatellifolia e Rhus natalensis têm um reconhecido valor nutricional e até hoje a planta inteira ou pelo menos partes destas têm sido utilizadas como plantas alimentares e/ou medicinais. Pimpinella anisum, além de ser usada na alimentação, têm um reconhecido valor medicinal. O milho (Zea mays) é de extraordinária relevância por fazer parte da dieta alimentar das populações da região, devido ao seu grande valor energético e riqueza em amido (Melo, 2008). $\mathrm{O}$ valor do milho como planta medicinal também foi muitas vezes referido em estudos realizados no país no tratamento de infecções urinárias, prevenção e tratamento de má nutrição, hipotensão, doenças musculares e ósseas, sendo ainda um excelente diurético (Costa \& Pedro, 2013). Estudos realizados no Brasil são coincidentes no que se refere ao uso de todos os órgãos da planta para fins medicinais, no tratamento da hipertensão, cistites e também no tratamento de doenças renais (Vendruscolo \& Mentz, 2006).

Finalmente, foi possível constatar que a infusão e decoção constituem as principais formas de preparação e utilização medicinal das plantas, o que pode estar ligado ao facto de que estas formas de preparação permitem várias aplicações dos extratos como medicamento. Relativamente aos órgãos usados destacam-se as folhas, raízes e, nalguns casos, principalmente nas herbáceas, o uso da planta 
inteira na preparação das soluções. Estas informações constituem uma questão preocupante, devido ao facto de que a remoção de raízes ou de plantas inteiras poderá a longo prazo levar à diminuição ou mesmo a extinção local de algumas destas espécies, se tivermos em conta a grande procura no uso de plantas medicinais que se verifica actualmente.

\section{Conclusão}

O presente estudo resume as principais informações contidas nos cadernos e notas de campo do colector JMD, no que se refere à utilização de plantas na Província da Huíla. O trabalho representa um significativo contributo para o conhecimento da flora da região, dos usos e costumes das populações que utilizam estas plantas como medicinais e para diversos fins. Grande parte dos dados aqui apresentados basearam-se nas informações prestadas pela população e por terapeutas tradicionais. Neste contexto, é encorajada a realização de trabalhos mais aprofundados e cientificamente sustentados que permitam a descoberta da composição química e dos princípios activos destas plantas visando a produção de medicamentos para o tratamento das várias doenças que afligem a nossa sociedade.

Anexo 1. Algumas espécies referidas no texto e respectivos sinónimos. A lista de sinónimos foi extraída da checklist de Plantas de Angola de Figueiredo \& Smith 2008.

\begin{tabular}{|l|l|}
\hline Nome científico & Sinónimo \\
\hline Acacia tristis (Welw. ex Oliv.) A.Schreib. & Acacia hebeclada DC. subsp. tristis DC. \\
\hline Adenia letouzeyi W.J. de Wilde & Adenia lobata Engl. \\
\hline Adenia rumicifolia Engl. \& Harms & $\begin{array}{l}\text { Adenia lobata (Jacq.) Engl. subsp. rumicifolia } \text { (Engl. \& Har- } \\
\text { ms) Lye }\end{array}$ \\
\hline $\begin{array}{l}\text { Amblygonocarpus andongensis (Oliv.) Exce- } \\
\text { ll \& Torre }\end{array}$ & Amblygonocarpus obtusangulus (Oliv.) Harms \\
\hline Blumea gariepina DC. & Blumea decurrens (Vahl.) Mersem \\
\hline Boerhavia adscendens Willd. & Boerhavia diffusa L. \\
\hline Carissa edulis (Forssk.) Vahl. & Carissa spinarum L. \\
\hline Chrysophyllum argyrophyllum Hiern & Englerophytum megalismontanum DC. \\
\hline Combretum polystictum Welw. ex Hiern & Combretum camporum Engl. \\
\hline $\begin{array}{l}\text { Crassocephalum multicorymbasum (Klatt) } \\
\text { S.Moore }\end{array}$ & Solanecio mannii (Hook.f) C.Jeffrey \\
\hline Eragrostis chalcantha Trin. & Eragrostis racemosa (Thunb.) Steud. \\
\hline Euphorbia rhipsaloides Welw. & Euphorbia tirucalli L. \\
\hline Fadogia monticola Robyns & Fadogia homblei De Wild. \\
\hline Ficus brevicula Hiern & Ficus pygmaea Welw. ex Hiern \\
\hline
\end{tabular}




\begin{tabular}{|c|c|}
\hline Ficus gnaphalocarpa (Miq.) C.C.Berg. & Ficus sycomorus $\mathrm{L}$. \\
\hline Ficus welwitschii Warb. & Ficus cordata Thunb. subsp. cordata Thunb. \\
\hline Gomphrena globulares L. & Gomphrena globosa L. \\
\hline Hannoa klaineana Pierre ex Engl. & Quassia undulata (Guill. \& Perr.) D.Dietr. \\
\hline Heeria argyrocrysea Engl \& Gilg. & Ozoroa argyrochrysea Engl. \& Gilg.)R.Fern. \& A.Fern: \\
\hline Helichrysum steetzii (Vatke) O.Hoffm. & Helichrysum kraussii Sch. \\
\hline Hymenodictyon kurria Hochst. & Hymenodictyon floribundum (Hochst. \& Steud.) Rob. \\
\hline Lefebverea welwitschii Engl. & Lefebverea grantii (Kingston ex Oliv.) \\
\hline Leucaena glauca Benth. & Leucaena leucocephala (Lam.) De Wit. \\
\hline Lippia asperifolia Rich. & Lippia javanica (Burm.f.) Spreng. \\
\hline Mimusops cuneifolia Baker & Manilkara obovata (Sabine \& G.Don) J.H.Hemsl. \\
\hline Ocimum viride Willd. & Ocimum gratissimum L. subsp. gratissimum L. \\
\hline Osyris abyssinica Hochst. \& A.Rich. & Osyris lanceolata Hochst. \& Steud. \\
\hline Picralima klaineana Welw. ex Hiern & Picralima nitida (Stapf) T.Durand \& H.Durand \\
\hline Pimpinella platyphylla Welw. & Pimpinella huillensis Welw. Ex Engl. \\
\hline Rhynchelytrum repens (Willd.) C.E.Hubb. & Melinis repens (Willd.) Zizka subsp. repens \\
\hline Vetiveria nigritana (Benth.) Stapf. & Chrysopogon nigritanus (Benth.) Veldkamp \\
\hline
\end{tabular}

\section{Agradecimentos}

Os autores agradecem à Direcção do ISCED Huíla pelo apoio prestado, à equipa do Herbário do Lubango pela colaboração no acesso às informações constantes nos cadernos de campo, e a José Camôngua Luís pela elaboração do mapa da área de estudo abrangida por este trabalho.

\section{Referências}

African Plants Database [http://www.ville-ge.ch/musinfo/bd/cjb/africa/].

Barbosa, L.A.G. (1970). Carta Fitogeográfica de Angola. Luanda, Instituto de Investigação Científica de Angola.

Bossard, E. (1996). La Medicine Traditionnelle au Centre et a L'Ouest de L'Angola. Lisboa, Instituto de Investigação Científica Tropical.

Chinsembu, K.C. (2016). Ethnobotanical study of medicinal flora utilised by traditional healers in the management of sexually transmitted infections in Sesheke District, Western Province, Zambia. B. Jour. Phar., http://dx.doi.org/10.1016/j.bjp.2015.07.030.

Chisingui, A.V., Gonçalves, F.M.P., Tchamba, J.J., Camôngua, J.L., Rafael, M.F.F \& Alexandre, J.L.M. (2018). Vegetation Survey of the Woodlands of Huíla Province. In: Climate change and adaptive land management in southern Africa - assessments, changes, challenges, and solutions (ed. by Revermann, R., Krewenka, K.M., Schmiedel, U., Olwoch, J.M., Helmschrot, J. \& Jürgens, N.), pp. 426-437, Biodiversity \& Ecology, 6, Klaus Hess Publishers, Göttingen \& Windhoek. doi:10.7809/b-e.00355 
Costa, E. (2012). Conhecimento tradicional e recursos terapêuticos naturais do Bengo, resultados do rastreio etnobotânico. Luanda, Centro de Investigação em Saúde em Angola.

Costa, E. \& Pedro, M. (2013). Plantas Medicinais de Angola. Luanda, Centro de Botânica da Universidade Agostinho Neto.

Cunningham, A.B. (1988). An Investigation of the herbal medicine trade in Natal/KwaZulu. Pietermaritzburg: Investigational Report No. 29, Institute of Natural Resources.

Figueiredo, E. \& Smith, G. (2008). Plants of Angola/Plantas de Angola. Pretoria, South African National Biodiversity Institute.

Figueiredo, E., Soares, M., Seibert, G., Smith, G.F. \& Faden, R.B. (2009). The botany of Cunene-Zambezi expedition with notes on Hugo Baum (1867-1950) Bothalia, 39(2)185-211.

Figueiredo E. \& Smith G. (2012). Common Names of Angolan Plants. Pretoria, Inhlaba Books.

Germishuizen, G., Meyer, N.L., Steenkamp, Y. \& Keith, M. (2006). A checklist of South African plants. Pretoria, Southern African Botanical Diversity Network.

Gonçalves, F.M.P. (2009). Contribuição para o conhecimento, distribuição e conservação da flora na Província da Huíla. Luanda, Tese de Licenciatura. DOI: 10.13140/RG.2.1.4006.0322.

Gossweiler, J. (1953). Nomes indígenas de plantas de Angola, Luanda. Agronomia Angolana No. 7.

INE. (2016). Resultados Definitivos: Recenseamento Geral da População e Habitação 2014. Luanda.

International Plant Names Index [http://www.ipni.org]

Kissanga, R. (2016). Valorização da flora de Cusseque e Caiundo no centro e sudeste de Angola e avaliação da biomassa lenhosa utilizada para combustível e construção. Lisboa, Tese de Mestrado.

Köppen, W. \& Geiger, R. (1936) Das geographische System der Klimate. Handbuch der Klimatologie (ed. by W. Köppen and R. Geiger), Vol 1 Part C pp. 1-44. Verlag von Gebrüder Borntraeger, Berlin.

Liengme, C.A (1983). A survey of ethnobotanical research in Southern Africa. Bothalia, 14(3 \& 4)621-629.

Lovett, J.C. (2018). Angola. African Journal of Ecology, 56:1-2

Maiato, F. \& Sweet, R.J. (2011). Guia de Pastos nas Áreas de Transumância. Luanda, GFA Consulting Group.

Maroyi, A. (2013). Traditional use of medicinal plants in south-central Zimbabwe: review and perspectives. Journal of Ethnobiology and Ethnomedicine, 9:31

Melo, R. (2008). Crenças, Poder e Práticas Medicinais Entre os Handa”, in Melo, Rosa (eds.) Para Lá da Manipulação dos Espíritos, crenças e práticas de cura entre os Handa no sul de Angola. Dakar, Série Monografias.

Mendonça, D.I.M.D., Mata, R.C.S., Sebastião, N.N., Borges, C.M.P. (2015). Um Breve Olhar Sobre Algumas Plantas Medicinais Angolanas. Química 138.

Ribeiro, A., Romeiras, M.M., Tavares, J., Faria, M.T. (2010). Ethnobotanical survey in Canhane village, district of Massingir, Mozambique: medicinal plants and traditional knowledge. Journal of Ethnobiology and Ethnomedicine, 6:33 
Teixeira, J.B. (1960) Apontamentos sobre plantas medicinais, aromáticas e resinosas, Separata da Agronomia Angolana 12:15-27.

The Plant List [http://www.theplantlist.org]

Vendruscolo, G.S. \& Mentz, L.A. (2006). Estudo da concordância das citações de uso e importância das espécies e famílias utilizadas como medicinais pela comunidade do bairro Ponta Grossa, Porto Alegre, RS, Brasil. Acta bot. bras. 20(2):367-382.

Van-Dúnem, M.B. (1979) Etat d'avancement de la recherche sur la médecine et la pharmacopée traditionnelles dans la République Populaire d'Angola, p. 197-203, Quatrième symposium interafricain OUA / CSTR sur la Pharmacopée traditionnelle et les plantes médicinales africaines. (Abidjan, Côte d'Ivoire, 25-29 septembre 1979)

Van-Dúnem, M.B. (1994) Plantas medicinais de Angola: medicamentos ao alcance de todos, 1a ed., Luanda: Cooperação Portuguesa, Embaixada de Portugal, 78 p.

Walters, W., Figueiredo, E., Crouch, N.R., Winter, P.J.D., Smith, G.F., Zemmermann, H.G. \& Mashope, B.K. (2011) Naturalised and invasive succulent of southern Africa. Abc Taxa 11.

WHO-World Health Organization (2003). Traditional medicine fact sheet No. 134, revised May 2003. http://www.who.int/mediacentre/factsheet/2003/fs134/en/.htm.

Data receção: $21 / 05 / 2018$

Data aprovação: 01/10/2018 